\title{
Proportion of patients in the Uganda rheumatic heart disease registry with advanced disease requiring urgent surgical interventions
}

\author{
WanZhu Zhang ${ }^{1}$, Emmy Okello ${ }^{1}$, Wilson Nyakoojo ${ }^{2}$, Peter Lwabi ${ }^{1}$, Charles K. Mondo ${ }^{3}$
}

1. Uganda Heart Institute, Cardiology

2. Uganda Heart Institute

3. Mulago Hospital, Medicine, Division of Cardiology

\begin{abstract}
:
Introduction: Since the establishment of the Uganda Rheumatic Heart Registry, over 900 patients have been enrolled. We sought to stratify the patients in the registry according to disease severity and optimal management strategy.

Methods: We reviewed data of 618 patients who had enrolled in the Registry between March 2010 and February 2013. The 67 patients who had died were excluded leaving 551 patients who were recruited. The optimum management strategy was determined according to the 2012 European Society of Cardiology guidelines on the management of valvular heart disease. Results: Out of the 551 patient's records evaluated, 398 (72.3\%) required invasive intervention, with 332(60.3\%) patients requiring surgery and $66(12.0 \%)$ requiring percutaneous mitral commissurotomy (PMC). This leaves only $27.7 \%$ of patients who required only medical management. Currently, majority of the patients $(498,90.4 \%)$ in the registry are on medical treatment. Of the $60.3 \%$ requiring surgical intervention, only $8.0 \%$ (44 patients) underwent valvular surgery and 5(1.0\%) patients of the $66(12.0 \%)$ underwent PMC successfully.

Conclusion: There is a high proportion of patients with severe disease that require surgical treatment yet they cannot access this therapy due to absence of local expertise.

Keywords: Rheumatic Heart Disease, surgical intervention, percutaneuos intervention

DOI: http://dx.doi.org/10.4314/ahs.v15i4.17

Cite as: Zhang W, Okello E, Nyakoojo W, Lwabi P, Mondo CK. Proportion of patients in the Uganda rheumatic heart disease registry with advanced disease requiring urgent surgical interventions. Afri Health Sci. 2015;15(4):1182-8. bttp:// dx.doi.org/10.4314/abs.v15i4.17
\end{abstract}

\section{Background}

Rheumatic fever/rheumatic heart disease Rheumatic Fever / Rheumatic Heart Disease (RF/RHD) is a major health problem in Uganda. The estimated prevalence of RF/RHD detected by echocardiography screening among children in the general population attending primary school is $1.5 \% .^{1}$ About $50 \%$ of newly diagnosed rheumatic heart disease patients in Uganda present with complications, notably heart failure, pulmonary hypertension, infective endocarditis and atrial fibrillation., ${ }^{2,3}$ The level of non-adherence to secondary prophylaxis is significantly high (46\%), ${ }^{4}$ with high rates of RHD associated with overcrowding and unemployment. ${ }^{5}$

The RHD Registry was established at the Uganda Heart Institute (UHI) in 2010 to provide a central database for
Corresponding author:
Charles K. Mondo,
Mulago Hospital, Medicine,
Division of Cardiology
Email: charlesmondo2011@gmail.com

all patients diagnosed with RHD. The purpose of the registry is to ensure patients are given optimal clinical care within available resources through accurate diagnosis, scheduled and timely follow-up review visits and timely invasive intervention where possible. At enrollment, detailed information is recorded regarding the patient's clinical condition, risk factors and complications of the disease. Patients are then offered education about their disease and prognosis. To date, over 800 patients have been enrolled and are being followed up.

Although the current registry documents disease patterns, severity and complications, it does not characterize patients according to treatment needs, such as those requiring percutaneous intervention and surgery. It is therefore time consuming to identify patients who require specific interventions when the need arises. Thus, to improve the organization of the registry, we sought to characterize patients in the registry with respect to disease severity and the recommended optimal management plan according to applicable guidelines. 
Accordingly, the aim of this study was to group all patients in the registry according to the most optimal primary management strategy: medical management, closed valvuloplasty (for mitral stenosis), and valve surgery.

\section{Methods \\ Study design}

This was a retrospective study that reviewed patients in the Uganda RHD Registry enrolled between March 2010 and February 2013. All patients in the registry were eligible. The patients who had died were excluded. Most of the information required to establish the most optimal management strategy for a particular patient was available in the records. These included demographic features, symptoms, clinical examinations, 12-lead Rest Electrocardiography (ECG), Transthoracic Echocardiography (ECHO), laboratory results and treatment. Patients who had incomplete data were called back for further assessment. Informed consent was obtained from all patients. The key study variables (current, optimal and planned management options) included medical treatment, Percutaneous Mitral Commissurotomy (PMC), valve repair or replacement surgery and palliative care. The study was approved by the School of Medicine Research and Ethics Committee of the College of Health Sciences, Makerere University.

\section{Study setting: The Uganda Heart Institute}

The Uganda Heart Institute (UHI) is the national referral centre for treatment of cardiovascular diseases in Uganda. It is located at Mulago National Referral \& University teaching Hospital complex. All patients with complex heart disease in the country and neighbouring states like East D. R. Congo, Rwanda, Northern Tanzania and South Sudan are referred here for specialized management. Closed heart surgeries like patient ductus arteniosus (PDA) ligation and pericardiectomy have been routinely performed at the UHI since 1997. Open heart surgery at the UHI began in October 2007 with support from visiting teams comprising cardiologists, a cardiac surgeon, perfusionists, cardiac intensive care unit (ICU) nurses, cardiac anesthesiologists, fellows, biomedical engineers, and other staff from sponsoring institutions. During these open heart surgery camps, the visiting teams carry a number of sundries for use in the operations to offset the cost of the operations. Pre-operative surgical conferences are held, and the different cadres of visiting health personnel would work with their Ugandan counterparts enabling appropriate skills transfer. To date, there are only three resident
Ugandan cardiac surgeons and two cardiac anesthesiologists at the UHI. On average, the center performs only twenty-five open heart surgeries per year. ${ }^{6}$ Most operations are congenital heart disease. For the past eight years since the program started only three patients with rheumatic heart disease have been operated on by a visiting surgeon. The cardiac catheterization laboratory was established in 2012, with the first closed balloon valvuloplasty for RHD mitral stenosis being performed later that year by a visiting team from the USA. ${ }^{7}$ Similarly, the local interventional team of four cardiologists is not competent to perform the procedure alone. Only five procedures were done in one year. The minimum cost of one open heart operation in Uganda is US\$ 6,000 . This is for the uncomplicated congenital heart diseases. The per capita income in Uganda in 2013 was approximately US $\$ 600$ annually, ${ }^{8}$ with an estimated 19.5 percent of the 35 million Ugandans living on less than US $\$ 1.00$ per day. ${ }^{8}$

\section{Protocols of choosing optimal management strat- egy}

The protocols we followed to categorize patients for the optimal management strategy are extensively described in the ESC Guidelines: "Guidelines on the management of valvular heart disease (version 2012) The Joint Task Force on the Management of Valvular Heart Disease of the European Society of Cardiology (ESC) and the European Association for Cardio-Thoracic Surgery (EACTS)."

\section{Data managementand analysis}

Data was captured on hard copy Case Report Forms and then transferred to the electronic Epidata sheet, and subsequently to Stata for analysis. Continuous variables were summarized as mean ( \pm standard deviation) and median ( \pm inter quartile range), and are presented in tables. Categorical data was summarized using frequency and percentages, and results are presented in frequency tables and bar charts.

\section{Results}

We reviewed data of 686 patients in the Uganda RHD Registry. The 68 patients lost to follow up and $67 \mathrm{pa}-$ tients who died were excluded. Several patients were called back for echocardiography reassessment due to incomplete information in the registry database. A total of 551 patients were enrolled. Overall, females $(72.2 \%)$ predominated, with a younger median age of males than females (21 years vs. 31 years respectively). 
Patterns of different treatment modality of patients in Uganda RHD registry. Currently, the majority of patients 502 (91.1\%) in the Uganda RHD Registry are on medical treatment without any intervention - Table 1, first column. Only 49 (8.97\%) patients have undergone surgical interventions with $44(8.0 \%)$ of patients underwent valvular surgery and five (1.0\%) patients underwent successful Percutaneous Mitral Commissurotomy (PMC). Two patients were followed up without any intervention as their disease is mild and are asymptomatic; and two patients were under palliative care $(0.4 \%)$. Table $1,2^{\text {nd }}$ column shows the numbers of patients corresponding to the optimal management strategy. Overall, 398 (72.23\%) required surgical interventions, leaving only $153(27.77 \%)$ patients who deserve to be on medical management.

Patients who need intervention. Table 1, column 3 shows that $349(63.3 \%)$ patients are currently in need of urgent surgical intervention. Of these, $288(52.3 \%)$ require outright surgery and $61(11.1 \%)$ are suitable candidates for (PMC).

Table 1: Treatment patterns of RHD patient in the Registry

\begin{tabular}{|c|c|c|c|}
\hline $\begin{array}{c}\text { Current or Implemented } \\
\text { Treatment }\end{array}$ & \multicolumn{2}{|c|}{ Optimal Treatment } & $\begin{array}{c}\text { In Urgent Need of Surgical } \\
\text { Intervention } \\
\end{array}$ \\
\hline $\begin{array}{l}\text { Surgical } \\
\text { Interventions } \\
\qquad \quad \text { Surgery } \\
\text { - } \quad \text { PMC }\end{array}$ & $\begin{aligned} 49(8.97 \%) \\
\bullet \quad 44(7.99 \%) \\
\bullet \quad 5(0.91 \%)\end{aligned}$ & $\begin{array}{c}398(72.23 \%) \\
\text { - } 332(60.25 \\
\%) \\
\text { - } 66(11.98)\end{array}$ & $\begin{array}{cc}349(63.3 \%) \\
\text { - } \quad 288(52.3 \%) \\
-\quad 61(11.1 \%\end{array}$ \\
\hline $\begin{array}{l}\text { Non-surgical } \\
\text { management } \\
\text { - Medical } \\
\text { - Palliative } \\
\text { - } \quad \text { Follow Up }\end{array}$ & $\begin{aligned} & 502(91.11 \%) \\
& \bullet 498(90.3 \%) \\
& \bullet 2(0.36 \%) \\
& \bullet 2(0.36 \%)\end{aligned}$ & $\begin{array}{cc}153(27.77 \%) \\
-\quad 149(27 \%) \\
\cdot \quad 2(0.36 \%) \\
\cdot \quad 2(0.36 \%)\end{array}$ & \\
\hline $\begin{array}{l}\text { Total number of } \\
\text { patients }\end{array}$ & $551(100 \%)$ & $551(100 \%)$ & $349(63.3 \%)$ \\
\hline
\end{tabular}

Column 3 shows that 349 (63.3\%) of patients in the registry are currently in need of urgent surgical intervention. PMC, Percutaneous Mitral Commissurotomy.

After, adjusting for the 49 patients who have received surgical intervention, the proportion of patients who need intervention stands at 69\% (57\% for surgery and 12\% for PMC), figure 1.

Figure 2 groups patients according to type of interven- tion needed by age group. Mitral valve surgery is the most needed intervention $(190,54.4 \%)$ in all the age groups, followed by double valve repair or replacement (87, 24\%), PMC (61, 17.5\%) and aortic valve surgery $(11,3.2 \%)$.

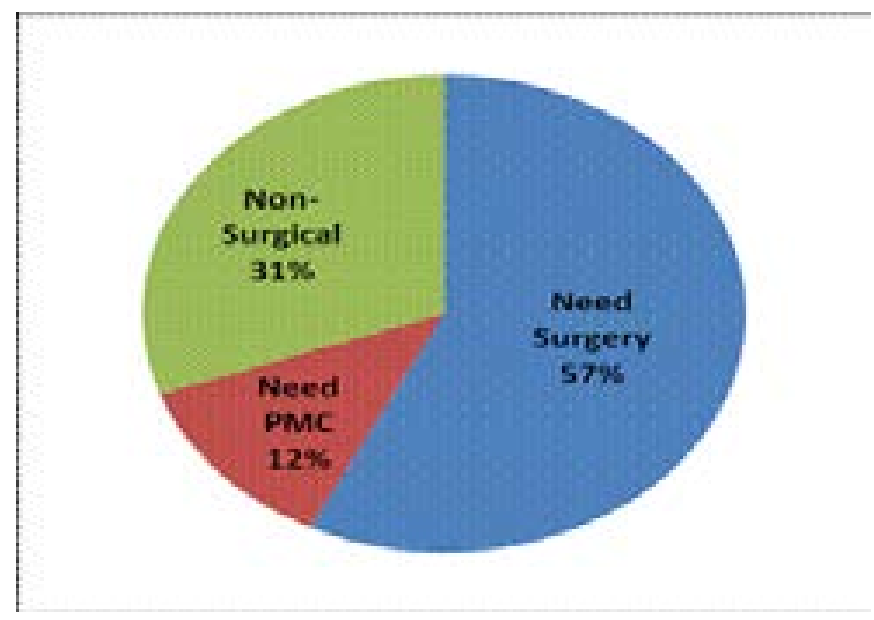

Figure 1. Proportion of patients who need intervention.

After removal of the 49 patients who received intervention, only $153(31 \%)$ patients require medical treatment, while $288(57 \%)$ require outright surgery and $61(12 \%)$ would benefit from PMC. 


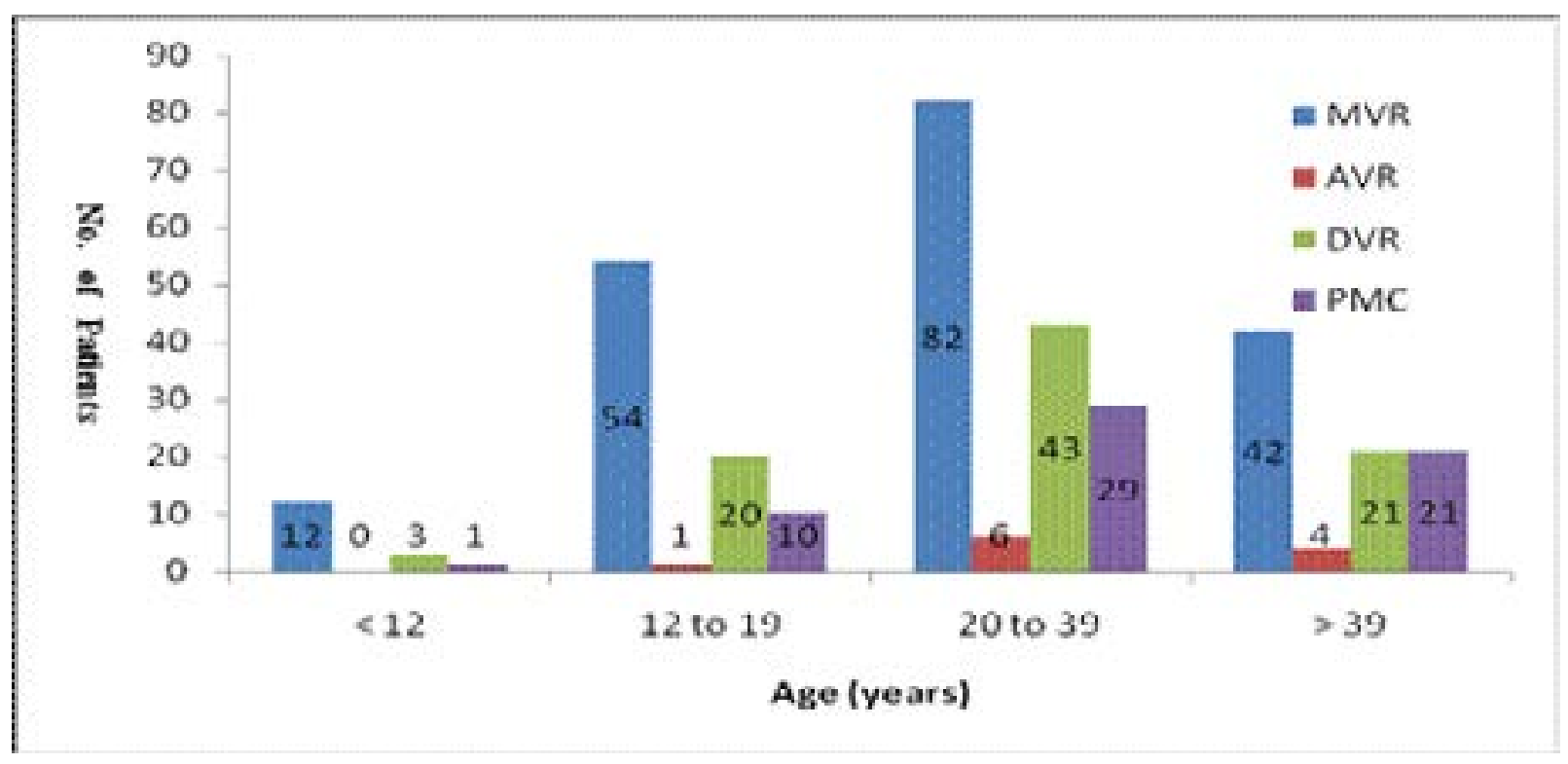

Figure 2. Patients who needs intervention in different age groups MVR, mitral valve replacement, AVR, aortic valve replacement, DVR, double valve replacement, PMC, percutanous mitral commisurotomy

Patients between 20 and 39 years accounted for the greatest number of patients in need of intervention $(160,45.8 \%)$, followed by patients of 40 years and above $(88,25.2 \%)$, patients between 12 to 19 years $(85$, $24.4 \%)$ and patients below 12 years $(16,4.6 \%)$. Table 2 shows the frequency of different types of valve lesions among patients who need surgical intervention. Out of 190 patients who need mitral valve surgery, Mitral Regurgitation (MR) $(139,73.5 \%)$ is the most frequent valvular lesion, followed by MR+MS (48, 25.4\%) and MS (three cases, 1.6\%).

Table 2. Patterns of valvular lesion in patients who needs intervention

\begin{tabular}{|c|c|c|}
\hline Valvular lesion & No. of patients & Totals \\
\hline & MVR & \multirow{4}{*}{190} \\
\hline MR & $139(73.5 \%)$ & \\
\hline MS & $3(1.6 \%)$ & \\
\hline \multirow[t]{2}{*}{$\mathrm{MR}+\mathrm{MS}$} & $48(25.4 \%)$ & \\
\hline & AVR & \\
\hline AR & $5(45.5 \%)$ & \multirow{3}{*}{11} \\
\hline AS & $2(18.2 \%)$ & \\
\hline \multirow[t]{2}{*}{$A R+A S$} & $4(36.4 \%)$ & \\
\hline & DVR & \\
\hline $\mathrm{MR}+\mathrm{AR}$ & $47(54.0 \%)$ & \multirow{8}{*}{87} \\
\hline $\mathrm{MR}+\mathrm{AS}$ & $3(3.4 \%)$ & \\
\hline $\mathrm{MS}+\mathrm{AR}$ & $5(5.7 \%)$ & \\
\hline $\mathrm{MS}+\mathrm{AS}$ & $3(3.4 \%)$ & \\
\hline$M R+A R+A S$ & $3(3.4 \%)$ & \\
\hline$M R+M S+A R$ & $14(16.1 \%)$ & \\
\hline$M S+A R+A S$ & $3(3.4 \%)$ & \\
\hline$M R+A S+A R+M S$ & $9(10.3 \%)$ & \\
\hline
\end{tabular}

Among 87 patients who need double valvular surgery, $\mathrm{MR}+\mathrm{AR}$ is the most frequent lesion (47, 54\%). 


\section{Discussion}

The main findings in this study are 1) there is a high proportion of patients that require urgent life-saving interventional procedures, and 2) there is a dire shortage and/or inability to access interventional services in this patient population. We have previously described the presenting features and clinical profile of RHD patients in this population. ${ }^{3}$ The late presentation, with advanced disease is multifactorial, including the inaccessibility to medicare and overcrowding, all of which are correlated with low socio-economic status. ${ }^{3}$

Patients that require urgent surgery. A large proportion of patients in the Uganda RHD Registry [288 (52.3\%)] required immediate surgery as the primary optimal management strategy, augmented with medical therapy. Although $61(11.1 \%)$ patients are suitable for PMC, the experience from the five patients who underwent PMC shows that many will require surgery anyway, as the procedure was successful in only two patients. These reported percentages may be underestimated as a significant proportion of patients were lost to follow-up. Invasive interventions in RHD have been reported by various investigators. ${ }^{9-12}$ However, these studies enrolled either very small numbers or report on specific interventions. Practically, these patients are on "palliative care," as it is unlikely that pharmacological management will improve their prognosis. ${ }^{13-15}$ Enrolment of these severe cases reflects the referral pattern to this site, which serves as the only tertiary care centre in Uganda and neighboring countries, but with capacity to handle a few cases. These patients are at high risk of developing $\mathrm{CHF}$ and infective endocarditis and will require surgical intervention. In the Heart of Soweto study, of 344 new cases of RHD seen at a tertiary care centre, $22 \%$ required valve replacement/repair within a year, and 26\% developed infective endocarditis within 30 months. ${ }^{16}$ In a four-year period $(2007$ - 2011), $45.5 \%$ of the 3,503 children 15 years of age and below with abnormal echocardiographs seen at the UHI had RHD, the majority with severe forms requiring surgery. ${ }^{17}$

Shortage of surgery services. The fact that only $37 \%$ patients in the registry received optimal treatment illustrates a necessity to improve care for patients by increasing access to intervention therapy in Uganda. The shortage cardiac surgery services in sub-saharan Africa has been comprehensively reviewed. ${ }^{18}$ The local team independently operates on average about four patients per year none of which is valvular surgery. ${ }^{6}$ Currently, valvular surgery and PMC are not done routinely at the UHI due to lack of expertise and high cost. Most patients have advanced diseases that require valve replacement. The minimum cost for single valvular replacement surgery if done in Uganda is US\$ 9,000; while the cost a PMC is US $\$ 5,000$. These figures exclude the cost of air tickets and lodging for the visiting team as there is no local capacity to do these procedures. This is not affordable to the affected patients as RHD is a disease of the poor and the majority of patients live on less than US\$ 1.0 per day. ${ }^{8}$ Relying on visiting surgeons and sponsors is not sustainable because the number of patients they can operate on during a brief visit is limited, and falls short of demand. Also, patients' conditions worsen day by day and many die before the next available visiting surgeon.

The intervention that is most needed is mitral valve repair or replacement surgery, which accounts for 190 cases $(54.4 \%)$. This is followed by double valve (mitral and aortic valves) surgery, PMC and aortic replacement. Unfortunately, sponsors are reluctant to invest in valvular surgery because of the associated long-term complications where patients may require a follow-up operation. Following the establishment of the cardiac catheterization services ${ }^{7}$ at UHI more than two years ago, only nine PMCs have been performed.

Since the establishment of Uganda RHD Registry four years ago, we prioritized health worker and patient education, disease surveillance, ${ }^{19}$ and Benzathine Penicillin secondary prophylaxis. We are continuing to conduct research to enable a deeper understanding of the pathogenesis of RHD and thus devise evidence based interventional approaches. ${ }^{20-22}$ Unfortunately, these are expensive endeavors which require governmental involvement and support, yet there is currently no government policy on the prevention and control of RF/ RHD in Uganda.

\section{Limitation of the study}

First, this is a retrospective data review study. The categorization of disease management relied on data created by a variety of doctors and nurses. For example, different doctors performed patients' ECHO reports. Second, there is no standardized national treatment guideline for management of patients who suffer from multiple valvular disease. The decision of optimal treatment for such patients in this study relied on investigators' experience. 


\section{Conclusion}

There is a high proportion of patients with advanced, severe RHD that require invasive treatment yet the service is not routinely available due to shortage of expertise. Many patients cannot access this therapy due to the high cost and the mandatory requirement to travel abroad. This calls for stakeholders to devise and prioritize mechanisms to treat the affected patients by developing local capacity.

\section{Acknowledgements.}

Research reported in this publication was supported by the Fogarty International Center, the National Heart Lung and Blood Institute, and the Common Fund of the National Institutes of Health under Award Number R24 TW008861. The content is solely the responsibility of the authors and does not necessarily represent the official views of the National Institutes of Health. The authors are grateful to the following persons for their invaluable support: Prof. Nelson Sewankambo, Prof. Moses R. Kamya, and the staff at the Uganda Heart Institute.

\section{References}

1. Andrea Beaton, Emmy Okello, Peter Lwabi, Charles Mondo, Robert McCarter and Craig Sable (2012). Echocardiography Screening for Rheumatic Heart Disease in Ugandan School children. Circulation. 125:3127-3132.

2. Emmy Okello, Zhang Wanzhu, Charles Musoke, Aliku Twalib, Barbara Kakande, Peter Lwabi , Nyakoojo B Wilson, Charles K. Mondo, Juergen Freers, R OdoiAdome (2013). Cardiovascular Complications in Newly Diagnosed Rheumatic Heart Disease Patients at Mulago Hospital, Uganda. Cardiovasc J Afr, 24: 82-87.

3. Zhang Wanzhu, Charles K Mondo, Emmy Okello, Charles Musoke, Barbara Kakande, Nyakoojo B Wilson, Juergen Freers (2013). Presenting Features of Newly Diagnosed Rheumatic Heart Disease Patients in Mulago Hospital - A pilot Study. Cardiovasc J Afr, 24: $28-33$.

4. Charles Musoke, Charles K Mondo, Emmy Okello, Zhang Wanzhu, Barbara Kakande, Nyakoojo B Wilson, Juergen Freers (2013). Benzathine Penicillin Adherence for Secondary Prophylaxis Among Patients Affected with Rheumatic Heart Disease Attending Mulago Hospital. . Cardiovasc J Afr; 24: 124-129

5. Emmy Okello, Charles K Mondo, Barbara Kakande, Elias Sebatta, Wilson B Nyakoojo, Richard OdoiAdome, Juergen Freers (2012). Socioeconomic and
Environmental Risk Factors Among Rheumatic Heart Disease Patients in Uganda. (PLoS ONE; 2012; 7(8): PubMed e43917. doi:10.1371/journal.pone.0043917).

6. Twalib O Aliku, Sulaiman Lubega, Peter Lwabi, Michael Oketcho, John O Omagino, Tom Mwambu. Outcome of patients undergoing open heart surgery at the Uganda heart institute, Mulago hospital complex. African Health Sciences Vol 14 Issue 4, December 2014, 946 - 953 PubMed.

7. Chris T. Longenecker, Emmy Okello, Peter Lwabi, Marco A. Costa, Daniel I. Simon, and Robert A. Salata. Management of Rheumatic Heart Disease in Uganda: The Emerging Epidemic of Non-AIDS Co-morbidity in Resource-Limited Settings. I Acquir Immune Defic Syndr. 2014 February 1; 65(2): e79-e80. doi:10.1097/ QAI.0b013e3182a03eb9.

8. TWB, (2014). "2013 Uganda GNI Per Capita". The World Bank (TWB). Retrieved 1 June 2015.

9. Vahanian A, Alfieri O, Andreotti F, Antunes MJ, Barón-Esquivias G, Baumgartner H, et al. Guidelines on the management of valvular heart disease (version 2012). European Heart Journal. [10.1093/eurheartj/ ehs109]. 2012;33(19):2451-96.

10. Erbel R, Aboyans V, Boileau C, Bossone E, Bartolomeo RD, Eggebrecht H, et al. 2014 ESC Guidelines on the diagnosis and treatment of aortic diseases. European Heart Journal. [10.1093/eurheartj/ehu281]. 2014;35(41):2873-926.

11. Hofer A, Woodland S, Reeve C. Mortality due to rheumatic heart disease in the Kimberley 2001-2010 (2014). Aust N Z J Public Health. 38: 139-41

12. Davies SB1, Hofer A, Reeve C. Mortality attributable to rheumatic heart disease in the Kimberley: a data linkage approach (2014). Intern Med J. 44: 1074-80.

13. Patience Olayinka Akinwusi; Johnson Olarewaju Peter; Adebayo Tolulope Oyedeji; Abiona Oluwadamilola Odeyemi (2013). The new face of rheumatic heart disease in South West Nigeria. International Journal of General Medicine. 6: 375-381

14. Tahereh Davarpasand and Ali Hosseinsabet (2014). Triple valve replacement for rheumatic heart disease: short- and mid-term survival in modern era. Interactive CardioVascular and Thoracic Surgery 1-6

15. Enriquez-Sarano M, Avierinos JF, Messika-Zeitoun D, Detaint D, Capps M, Nkomo V, Scott C, Schaff HV, Tajik AJ (2005). Quantitative determinants of the outcome of asymptomatic mitral regurgitation. $N$ Engl $J$ Med. 352: 875-883.

16. Karen Sliwa, Melinda Carrington, Bongani M. May- 
osi, Elias Zigiriadis, Robert Mvungi, and Simon Stewart (2010). Incidence and characteristics of newly diagnosed rheumatic heart disease in Urban African adults: insights from the Heart of Soweto Study. European Heart Journal. 31: 719-727.

17. Sulaiman Lubega, Twalib Aliku, Peter Lwabi. Echocardiographic pattern and severity of valve dysfunction in children with rheumatic heart disease seen at Uganda Heart Institute, Mulago hospital. African Health Sciences Vol 14 Issue 3, September 2014, 617 - 625 PubMed . 18. Charles Yankah1, Francis Fynn-Thompson, Manuel Antunes, Frank Edwin, Christine Yuko-Jowi, Shanthi Mendis, Habib Thameur, Andreas Urban, Ralph Bolman 3rd. Cardiac Surgery Capacity in Sub-Saharan Africa: Quo Vadis? Thorac Cardiovasc Surg. 2014 Aug;62(5):393401. doi: 10.1055/s-0034-1383723. Epub 2014 Jun 23. 19. Andrea Beaton, Emmy Okello, Twalib Aliku, Sulaiman Lubega, Peter Lwabi, Charles Mondo, Robert
McCarter, Craig Sable (2014). Latent Rheumatic Heart Disease: Outcomes Two Years after Echocardiographic Detection. Pediatr Cardiol. [Epub ahead of print]

20. Beaton A, Aliku T, Okello E, Lubega S, McCarter R, Lwabi P, Sable C (2014). The utility of handheld echocardiography for early diagnosis of rheumatic heart disease. J Am Soc Echocardiogr. 27: 42-49.

21. Okello E, Beaton A, Mondo CK, Kruszka P, Kiwanuka N, Odoi-Adome R, Freers J (2014). Rheumatic heart disease in Uganda: the association between MHC class II HLA DR alleles and disease: a case control study. BMC Cardiovasc Disord. 28: 14: 28.

22. Zühlke L, Engel ME, Karthikeyan G, Rangarajan S, Mackie P, Cupido B, et al. (2014). Characteristics, complications, and gaps in evidence-based interventions in rheumatic heart disease: the Global Rheumatic Heart Disease Registry (the REMEDY study). Eur Heart J. 2015 May 7;36(18):1115-22. doi: 10.1093/eurheartj/ ehu449. Epub 2014 Nov 25 